\title{
Non-evidence-Based Medicine: The Gastroenterologist's Role and Responsibility
}

\author{
Benjamin Lebwohl ${ }^{1}$
}

Published online: 26 February 2018

(c) Springer Science+Business Media, LLC, part of Springer Nature 2018

When I completed my training as a gastroenterologist-a long path starting with premedical curricular requirements, medical school, residency, and fellowship-I assumed that I was well prepared to take care of patients. I was therefore surprised to find that patients were asking me about conditions that were totally foreign to me: "Do I have a leaky gut?" "Should I be on a Candida diet?" Similarly, I found myself awash in unfamiliar terms when asking patients about their supplement use: Coenzyme Q10, black cohosh, apple cider vinegar, and countless agents I had never heard of. A search on PubMed would yield either no studies or studies suggesting a lack of effectiveness for the agent in question. It became apparent that I would need to develop a strategy, akin to the approach to the patient with abdominal pain or rectal bleeding: I would need to develop an approach to the patient who inquires about, or is heavily invested in, the category of treatments I call "non-evidence-based medicine."

Any physician with patient contact inevitably encounters unproven diagnostic techniques and therapeutic approaches, but gastroenterology is particularly replete with them. At any given moment, there are bestselling books vilifying a particular food group or ingredient or prescribing a diet to promote weight loss, counter inflammation, or cure or prevent diabetes, depression, arthralgias, fatigue, or a host of other maladies. Patients may present to the gastroenterologist with a stack of test results indicating various food intolerances based on unproven methodologies. The proliferation of such tests is a consequence of the current unsatisfactory state of affairs regarding the existing diagnostics and therapeutics for gastrointestinal disorders, particularly those of a functional nature. A patient with newly diagnosed irritable bowel syndrome, after having had a full evaluation

Benjamin Lebwohl

BL114@columbia.edu

1 Department of Medicine, Celiac Disease Center, Columbia University Medical Center, 180 Fort Washington Avenue, Suite 936, New York, NY 10032, USA for structural pathology, may feel frustrated that "none of the tests showed anything" and seek out testing outside of "mainstream" medicine in order to validate a basis for his/ her symptoms. The fact that the majority of patients with functional gastrointestinal disorders note a link between food and symptoms [1], coupled with the lack of proven diagnostic tools to identify specific triggers, permits the proliferation of unvalidated tests to fill the vacuum.

\section{What Gastroenterologists Should and Should Not Do}

One approach that does not suffice is for the gastroenterologist to shrug his or her shoulders and ignore the field entirely, paying attention only to evidence-based medicine and not addressing the questions or test results that come our way from these alternative sources. Just as we must learn how to incorporate new classes of medication into our practice as they are introduced and proven to be beneficial, we should also stay apprised of developments in the parallel world of non-evidence-based medicine, so as to help our patients who may be travelers in both worlds.

For instance, physicians should understand the implications of the Dietary Supplement Health and Education Act (DHEA) passed by Congress in 1994. This law established a parallel track for supplements that does not require that data be submitted to the Food and Drug Administration (FDA). In contrast to medications that undergo testing in multiple phases for safety and efficacy prior to their being made available to the public, supplements on this parallel track are presumed safe on introduction to the market, and the FDA initiates an investigation only if reports suggestive of a hazard are submitted. For example, the hepatotoxic effects of the nutritional supplement OxyELITE Pro were discovered not in a clinical trial but after more than 40 patients developed severe acute hepatitis, requiring liver transplantation in some cases [2]. 
The lower bar for safety required for supplements may be responsible for the increased incidence of supplement-induced harm in the USA. The rate of emergency department visits for adverse effects attributed to micronutrient supplements (excluding iron, calcium, and potassium) increased when comparing the time spans of 2004-2005 and 2013-2014 [3]. Supplement-induced liver failure has also increased over time, with proportion of drug-induced liver failure arising from supplements growing from $12.4 \%$ prior to 2008 to $21.1 \%$ from 2008 to 2015 [4].

It is also helpful for gastroenterologists to be familiar with specific prominent strains of thought within alternative medicine. For example, naturopathic medicine places an emphasis on prevention and incorporates nutritional and pharmaceutical approaches that emphasize the body's inherent self-healing process [5]. In contrast with the increasing specialization of conventional medicine, naturopathy emphasizes treating the whole person, with therapies designed to heal the physical, emotional, genetic, environmental, social, and spiritual dimensions [5]. This can be attractive to patients who feel let down by conventional medicine, particularly for those illnesses (such as functional gastrointestinal disorders) that are not easily cured using evidence-based medicine.

There are aspects of naturopathy that conventional practitioners may want to emulate, including a greater emphasis on preventive care. However, there are also aspects of naturopathy, practiced by some, though not all, practitioners, that range from the ineffective (e.g., homeopathy) to possibly harmful (e.g., intravenous chelation or vitamin infusions) to potentially hazardous to the individual and public health (e.g., vaccine refusal). One claims-based analysis in Washington State found that children who utilized naturopathic services were less likely to receive recommended vaccinations and more likely to develop a vaccine-preventable infection [6]. While naturopathy is not a monolith and is thus not tightly aligned to the anti-vaccine movement, it is troubling that in 2016, the Executive Director of the American Academy of Naturopathic Physicians stated that the organization's governing body is "discussing its stance on vaccinations" [7]. Beyond naturopathy, there is wider concern that patients who report using complementary and alternative medicines may be less likely to proceed with treatments proven to carry benefit; for example, among patients with breast cancer, use of complementary and alternative medicine was associated with decreased initiation of chemotherapy of proven efficacy [8].

\section{Gluten-Free Diets}

For gastroenterologists, avoidance of gluten among individuals without celiac disease is both a common scenario and a challenge as well as an opportunity to engage with patients

who are involved with non-evidence-based medicine. Interest in the gluten-free diet has exploded in recent years, as shown both in nationally representative surveys and internet searches [9, 10]. Although a subset of the population without celiac disease may develop symptoms triggered by gluten, and there are data suggestive of immune activation among that group [11], there are also a substantial number of individuals on a self-prescribed gluten-free diet who are actually reacting to fermentable oligo-, di-, monosaccharides, and polyols (FODMAPs) [12] or specifically to fructans [13]. It is also the case that a substantial number of people on a gluten-free diet have not been tested for celiac disease [14] and that upon evaluation approximately one-third of patients with a self-diagnosis of nonceliac gluten sensitivity are found to have another diagnosis in addition to, or instead of, their apparent adverse reaction to gluten; such diagnoses include intolerances to lactose or fructose, microscopic colitis, and small intestinal bacterial overgrowth [15].

Gastroenterologists should therefore take a careful history and evaluation of the patient on a self-prescribed glutenfree diet, as there is a strong possibility that such an assessment will be productive. Gastroenterologists should also be aware that strains of skepticism about vaccine safety are present in some gluten-avoidant individuals, and they should emphasize the public health benefits of vaccination and the harms of vaccine avoidance [16]. But ultimately, patients with nonceliac gluten sensitivity should be approached with humility given the uncertainty surrounding their condition. Uncertainty permeates scientific inquiry and medicine and should be acknowledged; this recognition of uncertainty is one of the qualities separating science from pseudoscience.

One practical way to address the patient asking about non-evidence medicine is to use a systematic approach, using the mnemonic SLEUTH (see Table 1). First, the clinician should identify what stage the patient is at regarding pursuing these strategies, as this may inform the focus of the conversation. A patient who is inquiring about taking probiotics for irritable bowel syndrome is different from a patient who is showing results of food intolerance tests that his naturopathic provider administered; the former might be guided toward a prudent path of trying one of the more commonly studied agents, while the latter might need to be educated about the lack of proven value of the testing that has been done. Lifestyle, particularly diet, is an important

Table 1 Approach to the patient who uses non-evidence-based medicine ("SLEUTH")
Stage
Lifestyle
Effective alternative
Underlying disease
Tablets
Harm 
aspect of the patient's health that should be elicited, since many non-evidence-based strategies include various dietary restrictions, some of which may be deleterious due to low fiber content. Clinicians should consider if an effective alternative for their patient's symptoms is available; a patient with Crohn's disease who has cut out gluten and casein for putative anti-inflammatory effects may be doing so for persistent symptoms, and may not have achieved full endoscopic and clinical remission and may benefit from a step up in therapy which may in turn yield superior longterm outcomes. Some patients pursuing non-evidence-based medicine may be doing so to treat an underlying disease that has been undiagnosed [15]. Clinicians should be queried specifically regarding the number of tablets or capsules they take, since it is common for patients following nonevidence-based practitioners to be prescribed upwards of 20 or 30 pills daily; after counting the total pill burden, patients may be open to the idea that there is a fair probability that at least one of their many supplements could carry an adverse effect, and may be amenable to efforts to reduce the quantity of supplement intake. More broadly, patients should be assessed for possible harm that non-evidence-based therapies can carry, such as hepatotoxic supplements, restrictive diets that can reactivate an eating disorder, or advice from practitioners to forgo immunizations for themselves or their children.

Ultimately, the approach should be individualized to the patient's circumstance, including his/her disease, symptoms, and attitude toward evidence-based and non-evidence-based medicine. We must be prepared to help our patients wherever they may be on their path, allying with them and keeping the dictum of "Do no harm" at the forefront.

\section{References}

1. Eswaran S, Tack J, Chey WD. Food: the forgotten factor in the irritable bowel syndrome. Gastroenterol Clin N Am. 2011;40:141-162.

2. Heidemann LA, Navarro VJ, Ahmad J, et al. Severe acute hepatocellular injury attributed to OxyELITE Pro: a case series. Dig Dis Sci. 2016;61:2741-2748.
3. Geller AI, Shehab N, Weidle NJ, et al. Emergency department visits for adverse events related to dietary supplements. $N$ Engl J Med. 2015;373:1531-1540.

4. Hillman L, Gottfried M, Whitsett M, et al. Clinical features and outcomes of complementary and alternative medicine induced acute liver failure and injury. Am J Gastroenterol. 2016;111:958-965.

5. American Association of Naturopathic Physicians. http://www. naturopathic.org/; Accessed 14.01.18.

6. Downey L, Tyree PT, Huebner CE, Lafferty WE. Pediatric vaccination and vaccine-preventable disease acquisition: associations with care by complementary and alternative medicine providers. Matern Child Health J. 2010;14:922-930.

7. Naturopaths, often derided as quacks, push to go mainstreamwith help from vitamin companies. https://www.statnews. com/2016/05/17/naturopaths-go-mainstream/; Accessed 14.01.18.

8. Greenlee H, Neugut AI, Falci L, et al. Association between complementary and alternative medicine use and breast cancer chemotherapy initiation: the breast cancer quality of care (BQUAL) study. JAMA Oncol. 2016;2:1170-1176.

9. Kim HS, Patel KG, Orosz E, et al. Time trends in the prevalence of celiac disease and gluten-free diet in the US population: results from the National Health and Nutrition Examination Surveys 2009-2014. JAMA Intern Med. 2016;176:1716-1717.

10. Laszkowska M, Shiwani H, Belluz J, et al. Socioeconomic vs health-related factors associated with google searches for glutenfree diet. Clin Gastroenterol Hepatol. 2017;16:295-297.

11. Uhde M, Ajamian M, Caio G, et al. Intestinal cell damage and systemic immune activation in individuals reporting sensitivity to wheat in the absence of coeliac disease. Gut. 2016;65:1930-1937.

12. Biesiekierski JR, Peters SL, Newnham ED, Rosella O, Muir JG, Gibson PR. No effects of gluten in patients with self-reported nonceliac gluten sensitivity after dietary reduction of fermentable, poorly absorbed, short-chain carbohydrates. Gastroenterology. 2013;145:e1-e3.

13. Skodje GI, Sarna VK, Minelle IH, et al. Fructan, rather than gluten, induces symptoms in patients with self-reported non-celiac gluten sensitivity. Gastroenterology. 2017;154:529-539.

14. Biesiekierski JR, Newnham ED, Shepherd SJ, Muir JG, Gibson PR. Characterization of adults with a self-diagnosis of nonceliac gluten sensitivity. Nutr Clin Pract. 2014;29:504-509.

15. Tavakkoli A, Lewis SK, Tennyson CA, Lebwohl B, Green PH. Characteristics of patients who avoid wheat and/or gluten in the absence of Celiac disease. Dig Dis Sci. 2014;59:1255-1261.

16. Rabinowitz LG, Zylberberg HM, Levinovitz A, Stockwell MS, Green PHR, Lebwohl B. Skepticism regarding vaccine and glutenfree food safety among patients with celiac disease and non-celiac gluten sensitivity. Dig Dis Sci. 2017;. https://doi.org/10.1007/ s10620-017-4879-1. 